\title{
Musculoskeletal Tumor Registry, Time to Act and Take Steps Forward
}

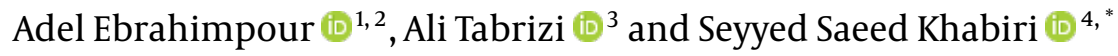 \\ ${ }^{1}$ Cancer Research Center, Shahid Beheshti University of Medical Sciences, Tehran, Iran \\ ${ }^{2}$ Physiotherapy Research Center, Shahid Beheshti University of Medical Sciences, Tehran, Iran \\ ${ }^{3}$ Department of Orthopedics, Imam Khomeini hospital, Urmia University of Medical Sciences, Urmia, Iran \\ ${ }^{4}$ Department of Orthopedic Surgery, Shohada Tajrish Hospital, Shahid Beheshti University of Medical Sciences, Tehran, Iran \\ "Corresponding author: Orthopedic Surgery Department, Shohada Tajrish Hospital, Shahid Beheshti University of Medical Science, Tehran, Iran. Email: \\ saeed.khabiri@gmail.com \\ Received 2021 September 14; Revised 2021 October 27; Accepted 2021 November 28.
}

Keywords: Musculoskeletal Tumor, Sarcoma, Disease Registry, Iran

\section{Dear Editor,}

Musculoskeletal tumors are rare, accounting for only 0.3 percent of all cancers at any age (1). The annual incidence of primary malignant bone tumors is 9 individuals per million, with an estimated mortality rate of 4 people per million (2). Raising public awareness about the epidemiology of these cancers will help prevent and treat health conditions. With the advancement of medical science and treatment methods, patients' prognosis and life expectancy have increased, but how much disability remains is an important issue (3). The appropriate management of these are different than non-musculoskeletal tumors because of difficulty in radiologic and histologic based diagnosing, requiring complex and demanding surgery (reconstructive surgery) with the involvement of various surgical specialties, and the need for adjuvant therapy. Since these tumors are rare, research to evaluate all aspects of diagnosis, treatment, and outcome are difficult and time-consuming. As a result, registries or national/regional cohort studies are the most practical tools for this survey. Armstrong et al. said, "The cancer registry is essential to cancer control, and cancer control is essential to (the survival of) the cancer registry" (4).

The cancer institute of Tehran University of Medical Sciences, Iran made the first attempt to register chronic diseases in Iran in 1955 (5). Etemadi et al. published the first data from the cancers registration system in Iran in 1956 (6). Cancer Research Center of Shahid Beheshti University of Medical Sciences is one of the best Iranin institutions for malignancy research and registration. An extensive study has been done to record musculoskeletal malignancies and comprehensive research projects in this field, and preliminary data from these research projects have been published. The study of Ewing sarcoma was performed at this center from 2008 to 2015, based on the Iranian National Cancer Registry (INCR) (7). Also, in a published study of soft tissue sarcomas based on INCR, the age-standardized incidence rate (ASIR) of total soft tissue sarcomas was 6.34 per million person-years (8). Regarding the epidemiology of spinal malignancies, 122 patients with a definite pathological diagnosis of chordoma were registered in this center (9).

These studies were among the first ones that use the national registration system to study musculoskeletal malignancy. In addition, they will help the future healthcare system plan for terms of controlling, preventing, and treating musculoskeletal malignancies. This information aids in visualizing tumor epidemic information. Despite the importance of this information, it appears that data collecting has remained unchanged in recent years, and it is still based on pathology. Given the importance of determining quality-adjusted life years (QALYs) and disability-adjusted life years (DALYs) and prognosis in patients, it appears that it is time to move on to the next step in gathering tumor data. In the population/community-based mechanism, the possibility of access to disease progression, treatments performed, complications, and patient function and the outcome can be measured (10). German Interdisciplinary Sarcoma Registry(GISAR), Musculoskeletal Tumor Registry (MsTR), The Scandinavian Sarcoma Group (SSG) Registry of soft tissue and bone tumors, bone and soft tissue tumors (BSTT) Registry have done qualitative registry in Japan . These registries contain population-based data, which allows us to gain a better understanding of all aspects of cancer management. Also, since some authors attribute disparities in sarcoma epidemiology to ethnicity and genetic factors $(11,12)$, actions should be taken to join and use a gene-based registry that serves as a valuable resource for 
expanding our understanding of cancer $(13,14)$.

In order to maximize the benefits of the chronic disease registry system, it is critical to investigate the flaws and improve data collection based on imaging and pathology findings. We should generalize our efforts to research and production in the field of new drugs production, manufacturing implants and prostheses, and modern radiotherapy technologies in our region. As mentioned above, there is currently a little research on the epidemiology of musculoskeletal tumors in Iran; despite enough cases and reliable results, there is no mention of survival and participant outcomes. Given the importance of dealing with tumors at the national level, good progress is currently being made in Iran. It has also created conception readiness for the multidisciplinary management doctrine. Comprehensive cancer centers are also being created and established across the country so that this platform may centrally launch a high-quality musculoskeletal tumor registry. According to our literature review, there is no musculoskeletal tumor registry in the MENA region (the Middle East and North Africa); additionally, because ethnicity and race are comparable, we can have a point of view on our region. Moreover, it can differ from other parts of East Asia, Europe, and the United States and be drawn based on differences, policies, and priorities. Due to the rarity and complexity of diagnosing and treating musculoskeletal tumors, it is critical to collect accurate and high-quality data to develop goals and strategies, which must then be reviewed and re-evaluated.

Finally, given the foregoing, it is necessary to have a population-based registration framework in which all "patient" information is included. In addition, the patient's histologic diagnosis and histogenetic typing, management, chemotherapy and radiation regimens, and procedures should all be documented. It should also include periodic follow-up in terms of therapy and surgical complications, recurrence, and mortality rate.

\section{Footnotes}

Authors' Contribution: Study concept and design, A. Eb.; Drafting of the manuscript, A. Ta., S. S. Kh.; Critical revision of the manuscript for important intellectual content, A. Ta.; Administrative, technical, and material support, S. S. Kh.; Study supervision, A. Eb.

Conflict of Interests: The authors mention that there is no conflict of interest in this study, and no benefits in any form have been or will be received from a commercial party related directly or indirectly to the subject of this manuscript.
Funding/Support: It was not declare by the authors.

\section{References}

1. Bergovec M, Kubat O, Smerdelj M, Seiwerth S, Bonevski A, Orlic D. Epidemiology of musculoskeletal tumors in a national referral orthopedic department. A study of 3482 cases. Cancer Epidemiol. 2015;39(3):298-302. doi: 10.1016/j.canep.2015.01.015. [PubMed: 25703268].

2. Bramer JA, Somford MP. (i) The epidemiology of primary skeletal malignancy. Orthop Trauma. 2010;24(4):247-51. doi: 10.1016/j.mporth.2010.06.006.

3. Fukushima T, Ogura K, Akiyama T, Takeshita K, Kawai A. Descriptive epidemiology and outcomes of bone sarcomas in adolescent and young adult patients in Japan. BMC Musculoskelet Disord. 2018;19(1):297. doi: 10.1186/s12891-018-2217-1. [PubMed: 30121085]. [PubMed Central: PMC6098838].

4. Armstrong BK. The role of the cancer registry in cancer control. Cancer Causes Control. 1992;3(6):569-79. doi: 10.1007/BF00052754. [PubMed: 1384732].

5. Ali Mohagheghi M, Mosavi-Jarrahi A. Review of cancer registration and cancer data in Iran, a historical prospect. Asian Pac J Cancer Prev. 2010;11(4):1155-7. [PubMed: 21133641].

6. Etemadi A, Sadjadi A, Semnani S, Nouraie SM, Khademi H, Bahadori M. Cancer registry in Iran: A brief overview. Arch Iran Med. 2008;11(5):57780. [PubMed: 18759534].

7. Ebrahimpour A, Chehrassan M, Sadighi M, Azizmohammad Looha M, Karimi A, Akbari A, et al. The Survival and Incidence Rate of Ewing Sarcoma; a National Population-based Study in Iran (2008-2015). Arch Bone Jt Surg. 2020;8(3):391-9. doi:10.22038/abjs.2020.44095.2206. [PubMed: 32766398]. [PubMed Central: PMC7358229].

8. Ebrahimpour A, Chehrassan M, Sadighi M, Azizmohammad Looha M, Karimi A, Raeisi A, et al. Mobile Spine Osseous Sarcoma: Descriptive Epidemiological Analysis Based on a National Population-Based Study. Arch Iran Med. 2021;24(8):629-35. doi: 10.34172/aim.2021.89. [PubMed: 34488331].

9. Chehrassan M, Ebrahimpour A, Sadighi M, Looha MA, Rad SB, Akbari ME. Epidemiologic trend of mobile spine and sacrum chordoma: A National population-based study. J Craniovertebr Junction Spine. 2020;11(3):226-31. doi: 10.4103/jcvjs.JCVJS_109_20. [PubMed: 33100773]. [PubMed Central: PMC7546048].

10. Sheikhtaheri A, Nahvijou A, Sedighi Z, Hadji M, Golmahi M, Roshandel G, et al. Development of a tool for comprehensive evaluation of population-based cancer registries. Int J Med Inform. 2018;117:26-32. doi: 10.1016/j.ijmedinf.2018.06.006. [PubMed:30032962].

11. Lupo PJ, Spector LG, O'Brien S, Schiffman JD, Hettmer S. Epidemiology of Bone and Soft Tissue Sarcomas. In: Arndt CA, editor. Sarcomas of Bone and Soft Tissues in Children and Adolescents. New York, USA: Springer Cham; 2021. p. 1-16. doi:10.1007/978-3-030-51160-9_1.

12. Diessner BJ, Weigel BJ, Murugan P, Zhang L, Poynter JN, Spector LG. Racial and Ethnic Differences in Sarcoma Incidence Are Independent of Census-Tract Socioeconomic Status. Cancer Epidemiol Biomarkers Prev. 2020;29(11):2141-8. doi: 10.1158/1055-9965.EPI-20-0520. [PubMed: 32928933]. [PubMed Central: PMC7641997].

13. Benna C, Simioni A, Pasquali S, De Boni D, Rajendran S, Spiro G, et al. Genetic susceptibility to bone and soft tissue sarcomas: A field synopsis and meta-analysis. Oncotarget.2018;9(26):18607-26. doi: 10.18632/oncotarget.24719. [PubMed: 29719630]. [PubMed Central: PMC5915097].

14. Diessner BJ.Associations of Social and Genetic Factors with Sarcoma Incidence and Outcomes [dissertation]. Minnesota, USA: University of Minnesota; 2020. 